\title{
Subsets of microsatellite-unstable colorectal cancers exhibit discordance between the CpG island methylator phenotype and $M L H 1$ methylation status
}

Jung H Kim ${ }^{1,4}$, Ye-Y Rhee ${ }^{1,4}$, Jeong-M Bae ${ }^{1}$, Hyeong-J Kwon ${ }^{1}$, Nam-Y Cho ${ }^{2}$, Mi J Kim ${ }^{3}$ and Gyeong H Kang ${ }^{1,2}$

${ }^{1}$ Department of Pathology, Seoul National University College of Medicine, Seoul, South Korea; ${ }^{2}$ Laboratory of Epigenetics, Cancer Research Institute, Seoul National University College of Medicine, Seoul, South Korea and ${ }^{3}$ Department of Diagnostic Pathology, Asan Medical Center, Seoul, South Korea

Although the presence of $M L H 1$ methylation in microsatellite-unstable colorectal cancer generally indicates involvement of the CpG island methylator phenotype (CIMP) in the development of the tumor, these two conditions do not always correlate. A minority of microsatellite-unstable colorectal cancers exhibit discordance between CIMP and MLH1 methylation statuses. However, the clinicopathological features of such microsatellite-unstable colorectal cancers with discrepant MLH1 methylation and CIMP statuses remain poorly studied. Microsatellite-unstable colorectal cancers $(n=220)$ were analyzed for CIMP and MLH1 methylation statuses using the MethyLight assay. Based on the combinatorial CIMP and MLH1 methylation statuses, the microsatellite-unstable colorectal cancers were grouped into four subtypes (CIMP-high (CIMP-H) MLH1 methylation-positive (MLH1m +), CIMP-H MLH1 methylation-negative, CIMP-low/0 (CIMP-L/0) MLH1m +, and CIMP-L/0 MLH1 methylation-negative), which were compared in terms of their associations with clinicopathological and molecular features. The CIMP-L/0 MLH1 methylation-negative and CIMP-H MLH1m + subtypes were predominant, comprising 63.6 and $24.1 \%$ of total microsatellite-unstable colorectal cancers, respectively. The discordant subtypes, CIMP-H MLH1 methylation-negative and CIMP-L/0 MLH1m +, were found in 5 and $7 \%$ of microsatellite-unstable colorectal cancers, respectively. The CIMP-H MLH1 methylation-negative subtype exhibited elevated incidence rates in male patients and was associated with larger tumor size, more frequent loss of MSH2 expression, increased frequency of KRAS mutation, and advanced cancer stage. The CIMP-L/0 MLH1m + subtype was associated with onset at an earlier age, a predominance of MLH1 loss, and earlier cancer stage. None of the CIMP-L/0 MLH1m + subtype patients succumbed to death during the follow-up. Our findings suggest that the discordant subtypes of colorectal cancers exhibit distinct clinicopathological and molecular features, although the proportion of discordant subtypes is low. The microsatellite-unstable colorectal cancers of the same CIMP status tended to exhibit different clinicopathological features depending on MLH1 methylation status.

Modern Pathology (2013) 26, 1013-1022; doi:10.1038/modpathol.2012.241; published online 1 February 2013

Keywords: colorectal cancer; $\mathrm{CpG}$ island; $\mathrm{CpG}$ island methylator phenotype; DNA methylation; MLH1

Colorectal cancer is the third most common cancer and the third leading cause of cancer deaths in the USA, independent of gender. ${ }^{1}$ In Korea, the

Correspondence: Professor GH Kang, MD, Department of Pathology, Seoul National University College of Medicine, 28 Yongondong, Chongno-gu, Seoul 110-744, South Korea.

E-mail: ghkang@snu.ac.kr

${ }^{4}$ These authors contributed equally to this work.

Received 18 September 2012; revised 11 December 2012; accepted

11 December 2012; published online 1 February 2013 incidence of colorectal cancer has rapidly increased during the past decade (1999-2008), becoming the second and fourth most common cancer in Korean men and women, respectively (http: //www.cancer.go.kr/). Colorectal cancer is a heterogeneous disease in terms of molecular carcinogenesis, and at least three molecular pathways are involved in colorectal carcinogenesis, including chromosomal instability, microsatellite instability, and the $\mathrm{CpG}$ island methylator phenotype (CIMP) pathway. ${ }^{2}$ Chromosomal instability is 
involved in the tumorigenesis and progression of the vast majority of the cancers occurring in the left large bowel (distal to the splenic flexure), whereas for cancers occurring in the right large bowel, chromosomal instability, microsatellite instability, and CIMP have important roles in tumorigenesis and tumor progression. ${ }^{3,4}$ Defects of the mismatch repair enzymes, including MLH1 and MSH2, generate microsatellite instability, which acts as the driving force for the tumorigenesis and progression observed in Lynch syndrome tumors. Apart from genetic mutations, promoter $\mathrm{CpG}$ island hypermethylation can inactivate $M L H 1$, which is causally associated with sporadic microsatelliteunstable colorectal cancers. In contrast with the germline mutations of MLH1 or MSH2, which lead to the development of microsatellite-unstable conventional adenomas, ${ }^{5-7}$ MLH1 promoter CpG island methylation is believed to occur during the progression of sessile serrated adenomas along the serrated neoplasia pathway., ${ }^{4,8-10}$ Thus, MLH1 promoter $\mathrm{CpG}$ island methylation is believed to act not as an initiator but as a modifier during molecular carcinogenesis. As MLH1 methylation is generally accompanied by concordant methylation of multiple CpG island loci, ${ }^{11,12}$ MLH1 methylation-positive $(\mathrm{MLH} 1 \mathrm{~m}+)$ tumors tend to also be CIMP-high (CIMP-H) microsatellite-unstable tumors.

As CIMP-H microsatellite-unstable colorectal cancers harbor genes that are inactivated not only by microsatellite instability but also by CIMP, the repertoire of inactivated genes in these colorectal cancers is likely to be distinct from (and possibly more extensive than) that exhibited by the CIMPlow/0 (CIMP-L/0) microsatellite-unstable colorectal cancers observed in Lynch syndrome. ${ }^{13}$ These differences in the repertoire of the inactivated genes between CIMP-H microsatellite-unstable colorectal cancers and CIMP-L/0 microsatelliteunstable colorectal cancers are expected to reflect the phenotypic differences between them. Jass, ${ }^{14}$ Jass et $a l^{15}$ and Young et $a l^{16}$ were the first to notify and to report the differences between these cancers. ${ }^{14-16}$ Several studies have since focused on and addressed this issue, ${ }^{17-20}$ showing that CIMP-H microsatellite-unstable colorectal cancers exhibit increased incidence rates in female patients, increased localization to the proximal large bowel, later age of onset, more frequent poor differentiation, increased frequency of $B R A F$ mutation, increased nodal metastasis, and shortened survival time.

Our previous study conducted to identify prognostic factors in microsatellite-unstable colorectal cancers found that CIMP-H status, BRAF mutation, poor differentiation, and advanced cancer stage were indices of poor prognosis in patients with microsatellite-unstable colorectal cancers. ${ }^{17}$ In that study, we found that all of the microsatelliteunstable colorectal cancers with MLH1 methylation examined were not CIMP-H, and furthermore, that all of the CIMP-H microsatellite-unstable colorectal cancers examined were not positive for MLH1 methylation. However, no study has reported the presence of CIMP-H microsatellite-unstable colorectal cancers without MLH1 methylation. Therefore, the clinicopathological features of this colorectal cancer subset remain unknown. Moreover, our understanding of the clinicopathological features of CIMP-L/0 microsatellite-unstable colorectal cancers with $M L H 1$ methylation remains limited. In this study, we classified microsatelliteunstable tumors into four subtypes depending on the combinatorial status of CIMP and MLH1 methylation and aimed to characterize the clinicopathological and molecular features of these four subtypes of microsatellite-unstable colorectal cancers.

\section{Materials and methods}

In all, 78 patients with microsatellite-unstable colorectal cancer(s) $(7.9 \%)$ were identified from a consecutive series of 989 patients who received curative surgery for colorectal cancers at Seoul National University Hospital (Seoul, South Korea) between 2004 and 2006. Six patients were excluded from the study because of the unavailability of paraffin-tissue blocks or a history of preoperative radiation. From a consecutive series of 2478 patients with colorectal cancer(s), who underwent curative surgery in Asan Medical Center (Seoul, South Korea), between 2004 and 2006, 170 patients $(7 \%)$ were found to have microsatellite-unstable colorectal cancer(s). Of these 170 cases, 148 cases were available for the molecular study. From the surgical files of the Department of Pathology, Seoul National University Hospital (Seoul, Korea) and of the Department of Diagnostic Pathology, Asan Medical Center (Seoul, Korea), we retrieved formalin-fixed, paraffin-embedded archival tissue blocks of microsatellite-unstable colorectal cancers. For patients with multiple microsatellite-unstable colorectal cancers, we analyzed individual tumors with advanced tumor staging or larger tumor size if individual tumors were of the same tumor stage. The electronic medical records were reviewed for the clinicopathological information of the 220 patients. Staging of the disease was classified according to the sixth edition of guidelines of the American Joint Committee on Cancer. This study was approved by the Institutional Review Board.

\section{Histological Assessment}

Through microscopic examination of hematoxylinand eosin-stained tissue glass slides, all of the cases were evaluated for the following histological parameters: tumor necrosis, papillary or villous appearance, acinar-form appearance, signet ring cell appearance, serrated appearance, eosinophilic cytoplasm, vesicular nuclei, extraglandular interstitial 
mucin, intraglandular dirty mucin, Crohn's-like lymphoid reaction, and intratumoral periglandular lymphoid cell infiltration. The defining criteria for these parameters were described previously. ${ }^{17}$ Medullary carcinoma was defined as a tumor that shows sheets of tumor cells with vesicular nuclei, prominent nucleoli, and abundant eosinophilic cytoplasm.

\section{DNA Isolation, the Analysis of KRAS and BRAF Mutations, and the Determination of Microsatellite Instability}

Under microscopic examination, the tumor areas that were most representative of the tumor histology and differentiation, as well as those that were most dense in tumor cellularity, were selected and marked. The marked areas were manually scraped with a scalpel blade and the scraped tissue samples were collected into $1.5 \mathrm{ml}$ microtubes containing $30 \mu \mathrm{l}$ tissue-lysis buffer (proteinase $\mathrm{K}(3 \mathrm{mg} / \mathrm{ml})$ and digestion solution (50 mM Tris, $1 \mathrm{mM}$ EDTA, $\mathrm{pH}$ 8.0, and 1\% Tween-20)). The samples were incubated at $55{ }^{\circ} \mathrm{C}$ for up to $48 \mathrm{~h}$. Following centrifugation, the supernatants were transferred into a newly labeled microtube. The samples were then placed into a $95^{\circ} \mathrm{C}$ heat block for $10 \mathrm{~min}$ to inactivate the proteinase $\mathrm{K}$. The mutational status of the KRAS codons 12 and 13 and BRAF codon 600 was assessed by PCR and sequence analysis as described previously. ${ }^{19,21}$ Microsatellite instability was assessed for the five NCI reference loci, including BAT25, BAT26, D2S123, D5S346, and D17S150. A tumor was classified as microsatellite-unstable or highlevel microsatellite instability when it exhibited microsatellite instability in at least two of the five microsatellite markers.

\section{CIMP Determination}

DNA samples were bisulfite-modified using the EZ DNA methylation kit (Zymo Research, Orange, CA, USA). The modified DNA samples were analyzed for their methylation status of eight CIMP-panel markers (CACNA1G, CDKN2A (P16), CRABP1, IGF2, MLH1, NEUROG1, RUNX3, and SOCS1) using a methylation-specific, probe-based, real-time PCR technology (the MethyLight assay). The DNA methylation of each examined marker was quantified and reported as a percent of methylated reference (PMR). PMR $=100 \times(($ methylated reaction/ALU)sample/(methylated reaction/ALU)M.SssI-reference). We considered a CpG island locus methylated when an exponential amplification curve was present and the PMR value was $>4$. The tumors were classified as CIMP-high (CIMP-H), CIMP-L, or CIMP-0 if the tumor exhibited methylation of $>4,1-4$, or no marker(s), respectively. MethyLight assay has been evaluated and validated for its precision and performance characteristics. ${ }^{22,23}$

\section{Immunohistochemistry}

Sections ( $5 \mu \mathrm{m}$ thick) were subjected to antigen retrieval in $10 \mathrm{mmol} / \mathrm{l}$ citrate buffer $(\mathrm{pH} \mathrm{6.0)}$ using microwave irradiation. After blocking with $3 \% \mathrm{H}_{2} \mathrm{O}_{2}$ and $10 \%$ normal goat serum, the sections were incubated with either a monoclonal anti-MSH2 antibody (Oncogene Science, Cambridge, MA, USA) or a monoclonal anti-hMLH1 (PharMingen, San Diego, CA, USA) for the immunohistochemical staining of the tumor cell nuclei. Nuclear expression of these proteins was classified as present or absent relative to the staining of non-neoplastic cell nuclei, which provided a positive internal control for each slide. The immunohistochemical evaluation was performed by two pathologists (JMB and MJK) who were blinded to the DNA methylation statuses of the cases.

\section{Statistics}

The statistical analyses were performed using the statistics program SPSS software (SPSS version 15.0, Chicago, IL, USA). All of the $P$-values were two-sided, and statistical significance was set at $P<0.05$. The $\chi^{2}$ test (or Fisher's exact test for categories with an $n$ value of $<10$ ) was performed on categorical data, and the two-tailed Student's $t$ test was used to evaluate the significance of the differences between two means. Disease-free survival was measured from the date of resection of the colorectal cancer to the date of recurrence, second primary colon cancer, or death from any cause, which occurred first, or to the date of the last clinical follow-up (before 31 May 2010). The disease-free survival rates were assessed with the Kaplan-Meier log-rank test. To control for confounding factors, we used the Cox proportionalhazards regression model to calculate the hazard ratio of death for each prognostic parameter found for the Kaplan-Meier log-rank tests.

\section{Results}

\section{Subsite Distribution of Microsatellite-Unstable Colorectal Cancers}

To identify whether the frequency of high-level microsatellite instability gradually changes along the large bowel from the cecum to the rectum or abruptly at the splenic flexure, we compared the frequency of high-level microsatellite instability according to bowel subsites. Tumors located in the cecum, ascending colon, transverse colon, or descending colon showed frequencies of high-level microsatellite instability $>17 \%$, whereas tumors located in sigmoid or rectum displayed frequencies 
of high-level microsatellite instability $<7 \%$ (Figure 1). No difference of high-level microsatellite instability frequency was noted between transverse colon and descending colon, but a significant difference of high-level microsatellite instability frequency was present between descending colon and sigmoid colon (17 vs 6\%, P-value $<0.001$, Fisher's exact test).

\section{Four Subtypes of Microsatellite-Unstable Colorectal Cancers}

We analyzed the methylation status of eight CIMPpanel markers using the MethyLight assay and determined the status of CIMP and MLH1 methylation of 220 cases of microsatellite-unstable tumors. Of the tumors examined, 29 and $31 \%$ of the cases were positive for CIMP and MLH1 methylation, respectively. In contrast to the general notion that CIMP-H microsatellite-unstable tumors are positive for MLH1 methylation, $12 \%(n=27)$ of the microsatellite-unstable cases exhibited discordant CIMP and MLH1 methylation status. Of the CIMP-H tumors $(n=64), 17.2 \%(n=11)$ were MLH1 methylation-negative (MLH1m-), whereas of the MLH1m + tumors $(n=69), 23.2 \% \quad(n=16)$ were CIMP-L/0. Based on the combinatorial statuses of CIMP and MLH1 methylation, the microsatelliteunstable tumors could be classified into the following four subtypes: CIMP-L/0 MLH1m - $(n=140$, $64 \%)$, CIMP-L/0 MLH1m $+(n=16,7 \%)$, CIMP-H MLH1m - $(n=11,5 \%)$, and CIMP-H MLH1m + $(n=53,24 \%)$ (Figures 2 and 3 ). To exclude the possibility that the absence of $M L H 1$ methylation in the CIMP-H MLH1m - subtype or the presence of MLH1 methylation in the CIMP-L/0 MLH1m + subtype of colorectal cancers might be attributed to technical errors, we performed the MethyLight analyses in triplicate using two different sets of MethyLight primers and probes that targeted the proximal and distal regions of MLH1 promoter. We found consistent results, thereby excluding the influence of technical errors in the MLH1 MethyLight analyses (data not shown).

\section{The Clinicopathological Features of Four Subtypes of Microsatellite-Unstable Colorectal Cancers}

The average age of the CIMP-L/0 MLH1m + subtype patients was 53.2 years, similar to that of the CIMPL/0 MLH1m - subtype patients (53.1 years). In contrast, the average age of the CIMP-H MLH1m subtype patients (average, 60.0 years) was higher than that of the CIMP-L/0 MLH1m - subtype patients but lower than that of the CIMP-H MLH1m + subtype patients (average, 66.2 years) $(P=0.104$ and 0.052 , Student's $t$-test, respectively) (Figure 4a). The male-to-female patient ratio was similar between the CIMP-L/0 MLH1m - and the CIMP-L/0 MLH1m + subtypes, whereas the male-to-

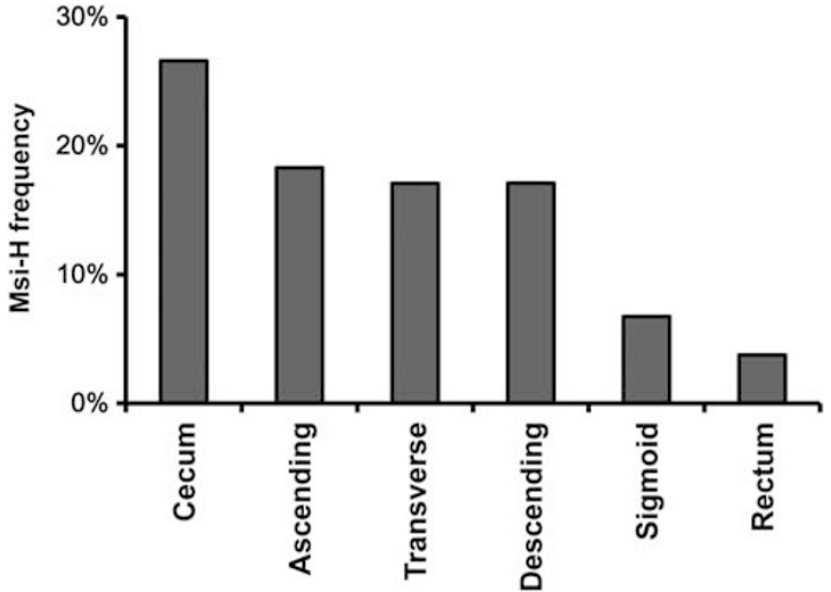

Figure 1 Frequencies of microsatellite instability-high in colorectal cancers along bowel subsites.

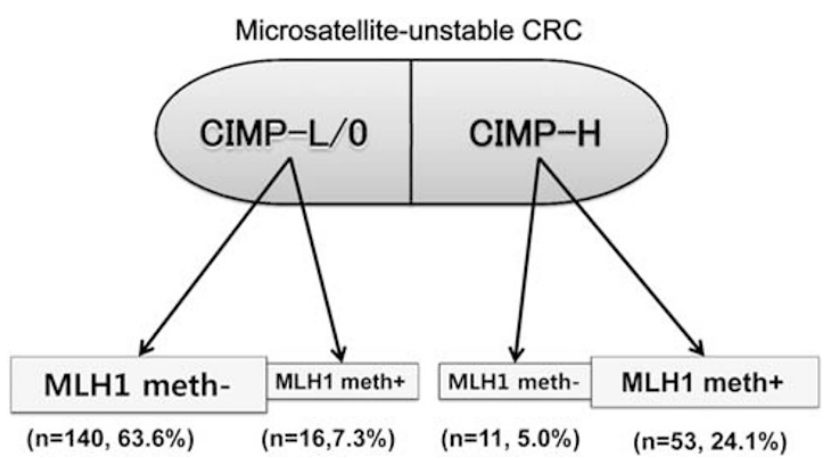

Figure 2 The four subtypes of microsatellite-unstable colorectal cancers according to the combinatorial statuses of $\mathrm{CpG}$ island methylator phenotype and MLH1 methylation.

female patient ratio of the CIMP-H MLH1msubtype was significantly different from that of the CIMP-H MLH1m + subtype (10:1 vs 24:29, $P=0.007$, Fisher's exact test, two sided) (Table 1). The preponderance of the right colon was more distinct in the CIMP-H MLH1m + and CIMP-H MLH1m - tumor subtypes than in the CIMP-L/0 MLH1m - and CIMP-L/0 MLH1m + tumor subtypes (85 and $73 \%$ vs 60 and $56 \%$, respectively, $P=0.009$, $\chi^{2}$ test). Multiple synchronous adenocarcinomas within the large bowel were detected at a frequency of $>10 \%$ in all of the subtypes except for the CIMPH MLH1m + subtype, which only gave rise to single cancers $\left(P=0.003, \chi^{2}\right.$ test). The CIMP-H MLH1m + and CIMP-H MLH1m - subtype tumors were larger than CIMP-L/0 MLH1m - and CIMP-L/0 MLH1m + tumors (Figure 4b). Poorly differentiated adenocarcinomas were more frequent in the CIMP-H MLH1m + and CIMP-H MLH1m - tumor subtypes (43 and $36 \%$, respectively) than in the CIMP-L/0 MLH1m + and CIMP-L/0 MLH1m - tumor subtypes (25 and $17 \%$, respectively) ( $P=0.005, \chi^{2}$ test). Nodal metastasis was more frequent in the CIMP-H MLH1m - $(46 \%)$ and the CIMP-H MLH1m + $(40 \%)$ tumor subtypes than in the CIMP-L/0 MLH1m - $(26 \%)$ and the CIMP-L/0 MLH1m + 


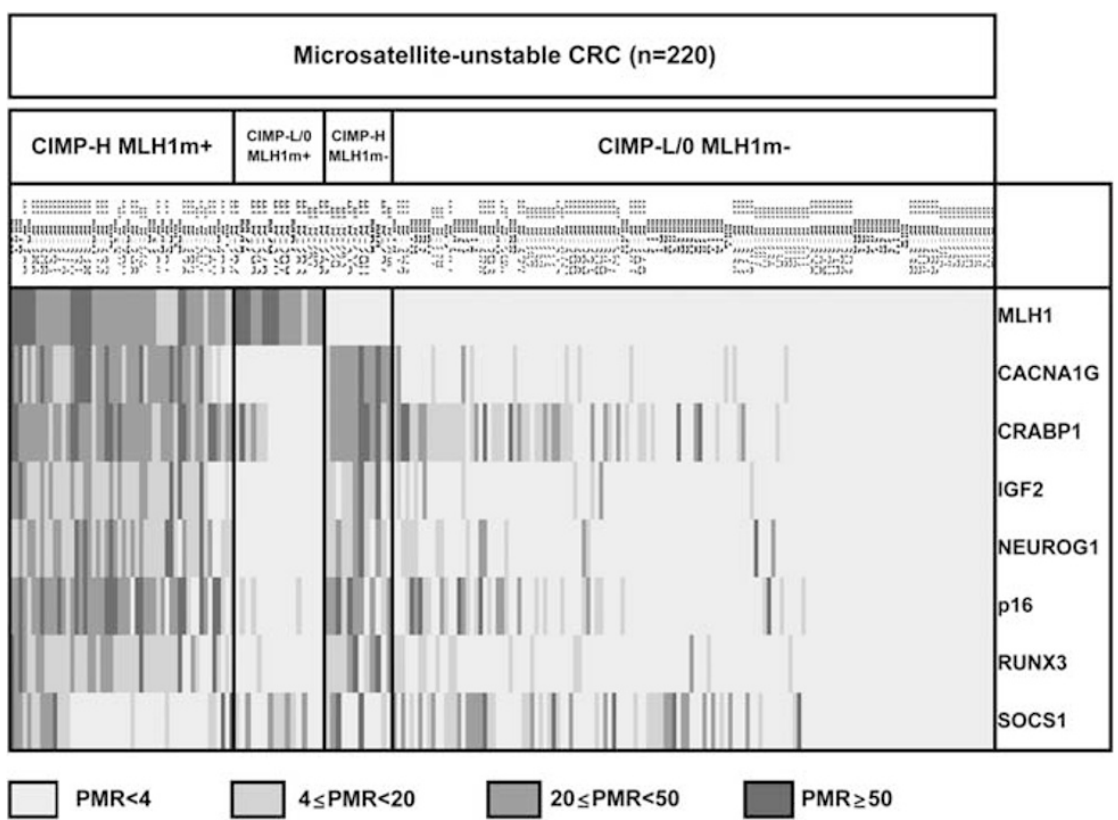

Figure 3 The methylation map of eight CpG island methylator phenotype (CIMP) markers for 220 microsatellite-unstable colorectal cancer samples.
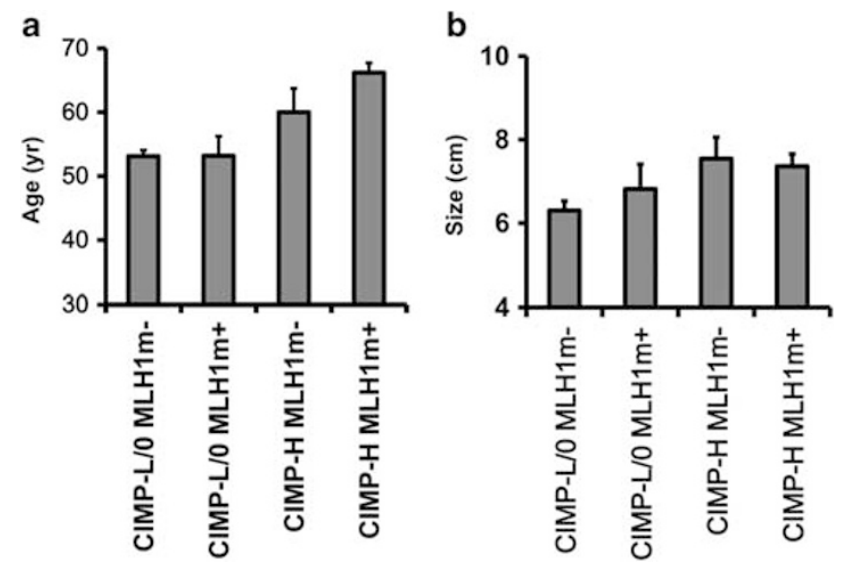

Figure 4 (a) The average age of the patients with each subtype of microsatellite-unstable colorectal cancer. (b) The average tumor size of the four subtypes of microsatellite-unstable colorectal cancer.

tumor subtypes (13\%) $\left(P=0.066, \chi^{2}\right.$ test). The CIMPH MLH1m + tumor subtype exhibited more frequent acinar form, and signet ring cell appearances but less frequent papillary appearances and stratified nuclei compared with the other three tumor subtypes. The frequency of medullary carcinoma was not different between the four subyptes.

\section{The Comparison of Molecular Features Between Four Colorectal Cancer Subtypes}

The BRAF mutation was detected at a frequency of $34 \%$ in the CIMP-H MLH1m + subtype tumors and at frequencies $<10 \%$ in the other three subtypes
$\left(P<0.001, \chi^{2}\right.$ test). In contrast, the KRAS mutation was detected in the CIMP-H MLH1m + subtype tumors at a frequency of $8 \%$, in contrast to the other three subtypes, which exhibited KRAS mutation frequencies of $>15 \%$. $\left(P=0.002, \chi^{2}\right.$ test $)$. In particular, the KRAS mutation was detected in the CIMPH MLH1m - subtype tumors at a frequency of $55 \%$. Immunohistochemical analyses revealed that the CIMP-H MLH1m - subtype tumors exhibited loss of MLH1 (27\%) and MSH2 (55\%), whereas the CIMP-H MLH1m + subtype tumors exhibited predominant loss of MLH1 (85\%) compared with MSH2 (9\%). For the CIMP-L/0 MLH1m - subtype tumors, the loss of MLH1 and MSH2 were found in 42 and $51 \%$ of the cases, respectively. In contrast, the CIMP-L/0 MLH1m + subtype tumors exhibited loss of MLH1 and MSH2 in 94 and $6 \%$ of the cases, respectively.

\section{Survival Analysis}

Kaplan-Meier analysis of DFS revealed that TNM stage, CIMP status, Crohn's-like lymphoid reaction, and histological differentiation represent statistically significant prognostic factors in patients with microsatellite-unstable colorectal cancer (Table 2). Furthermore, age, BRAF mutation, and peritumoral lymphocytic reaction were marginally significant factors. Those seven factors, which were identified to be significant or marginally significant parameters by Kaplan-Meier survival analysis, were included into the multivariate analysis that was performed using the Cox proportional hazard methods model. The TNM stage and CIMP status were found to be independent prognostic parameters. 
Table 1 Comparison of clinicopathological features between four molecular subtypes of microsatellite-unstable colorectal cancers

\begin{tabular}{|c|c|c|c|c|c|}
\hline & $\begin{array}{c}C I M P-L / 0 M L H 1 m- \\
(\mathrm{n}=140)\end{array}$ & $\begin{array}{c}C I M P-L / O M L H 1 m+ \\
(\mathrm{n}=16)\end{array}$ & $\begin{array}{c}C I M P-H \text { MLH1m - } \\
(\mathrm{n}=11)\end{array}$ & $\begin{array}{c}C I M P-H M L H 1 \mathrm{~m}+ \\
(\mathrm{n}=53)\end{array}$ & P-value \\
\hline \multicolumn{6}{|l|}{ Gender } \\
\hline Male & $90(64 \%)$ & $9(56 \%)$ & $10(91 \%)$ & $24(45 \%)$ & \multirow[t]{2}{*}{0.016} \\
\hline Female & $50(36 \%)$ & $7(44 \%)$ & $1(9 \%)$ & $29(55 \%)$ & \\
\hline \multicolumn{6}{|l|}{ Age (years) } \\
\hline$\leq 55$ & $83(59 \%)$ & $9(56 \%)$ & $5(45 \%)$ & $10(19 \%)$ & \multirow[t]{2}{*}{$<0.001$} \\
\hline$\geq 56$ & $57(41 \%)$ & $7(44 \%)$ & $6(55 \%)$ & $43(81 \%)$ & \\
\hline \multicolumn{6}{|l|}{ Location } \\
\hline Right colon & $84(60 \%)$ & $9(56 \%)$ & $8(73 \%)$ & 45 (85\%) & \multirow{3}{*}{0.018} \\
\hline Left colon & $28(20 \%)$ & $5(31 \%)$ & $3(27 \%)$ & $4(8 \%)$ & \\
\hline Rectum & $28(20 \%)$ & $2(13 \%)$ & 0 & $4(8 \%)$ & \\
\hline \multicolumn{6}{|l|}{ Gross type } \\
\hline Polypoid & $15(11 \%)$ & 0 & 0 & 0 & \multirow{3}{*}{0.088} \\
\hline Ulcerofungating & $112(80 \%)$ & $14(88 \%)$ & $11(100 \%)$ & 46 (87\%) & \\
\hline Ulceroinfiltrative & $13(9 \%)$ & $2(13 \%)$ & 0 & $7(13 \%)$ & \\
\hline \multicolumn{6}{|l|}{ Multiplicity } \\
\hline Single & $125(89 \%)$ & $12(75 \%)$ & $8(73 \%)$ & $53(100 \%)$ & \multirow[t]{2}{*}{0.003} \\
\hline Multiple & $15(11 \%)$ & $4(25 \%)$ & $3(27 \%)$ & 0 & \\
\hline \multicolumn{6}{|l|}{ Differentiation } \\
\hline Well & $15(11 \%)$ & 0 & $1(9 \%)$ & $1(2 \%)$ & \multirow{3}{*}{0.005} \\
\hline Moderate & $101(72 \%)$ & $12(75 \%)$ & $6(55 \%)$ & $29(55 \%)$ & \\
\hline Poor & $24(17 \%)$ & $4(25 \%)$ & $4(36 \%)$ & $23(43 \%)$ & \\
\hline \multicolumn{6}{|l|}{ Histological subtype } \\
\hline Medullary & $7(5 \%)$ & $2(13 \%)$ & $1(9 \%)$ & $6(11 \%)$ & \multirow{3}{*}{0.357} \\
\hline carcinoma & & & & & \\
\hline Non-medullary & $133(95 \%)$ & $14(88 \%)$ & $10(91 \%)$ & $47(89 \%)$ & \\
\hline \multicolumn{6}{|l|}{ T stage } \\
\hline T1 and 2 & $28(20 \%)$ & $1(6 \%)$ & $2(18 \%)$ & $3(6 \%)$ & \multirow[t]{2}{*}{0.066} \\
\hline T3 and 4 & $112(80 \%)$ & $15(94 \%)$ & $9(82 \%)$ & $50(94 \%)$ & \\
\hline \multicolumn{6}{|l|}{$N$ stage } \\
\hline No & $104(74 \%)$ & $14(88 \%)$ & $6(55 \%)$ & $32(60 \%)$ & \multirow[t]{2}{*}{0.105} \\
\hline $\mathrm{N} 1$ and 2 & $36(26 \%)$ & $2(13 \%)$ & $5(45 \%)$ & $21(40 \%)$ & \\
\hline \multicolumn{6}{|l|}{ M stage } \\
\hline Mo & $135(96 \%)$ & $16(100 \%)$ & $10(91 \%)$ & $51(96 \%)$ & \multirow[t]{2}{*}{0.673} \\
\hline M1 & $5(4 \%)$ & 0 & $1(9 \%)$ & $2(4 \%)$ & \\
\hline \multicolumn{6}{|l|}{$K R A S^{\mathrm{a}}$} \\
\hline Wild type & $110(81 \%)$ & $10(67 \%)$ & $5(45 \%)$ & $47(92 \%)$ & \multirow[t]{2}{*}{0.002} \\
\hline Mutant type & $26(19 \%)$ & $5(33 \%)$ & $6(55 \%)$ & $7(8 \%)$ & \\
\hline \multicolumn{6}{|l|}{$B R A F^{\mathrm{b}}$} \\
\hline Wild type & $134(96 \%)$ & $15(94 \%)$ & $10(91 \%)$ & $34(64 \%)$ & \multirow[t]{2}{*}{$<0.001$} \\
\hline Mutant type & $5(4 \%)$ & $1(6 \%)$ & $1(9 \%)$ & $19(36 \%)$ & \\
\hline Immunohistochemis & & & & & \\
\hline MLH1 loss & $56(42 \%)$ & $15(94 \%)$ & $3(27 \%)$ & $45(85 \%)$ & $<0.001$ \\
\hline MSH2 loss & $69(51 \%)$ & $1(6 \%)$ & $6(55 \%)$ & $5(9 \%)$ & \\
\hline Retained & $10(7 \%)$ & 0 & $2(18 \%)$ & $3(6 \%)$ & \\
\hline
\end{tabular}

${ }^{\mathrm{a}} \mathrm{KRAS}$ sequencing analysis failed for 109 cases.

$\mathrm{b}_{B R A F}$ mutant allele-specific PCR failed for one case.

When we analyzed the four tumor subtypes for their disease-free survival rates, we found that there was a significant difference in disease-free survival between the molecular subtypes: 5 -year disease-free survival, 94\% in the CIMP-L/0 MLH1m + subtype,
$89 \%$ in the CIMP-L/0 MLH1m - subtype, $73 \%$ in the CIMP-H MLH1m + subtype, and 60\% in the CIMP-H MLH1m - subtype $(P=0.0067$; Figure 5$)$. No case of the CIMP-L/0 MLH1m + subtype succumbed to death but one case developed tumor recurrence in the lung. 
Table 2 Univariate and multivariate analysis of disease-free survival in microsatellite-unstable colorectal carcinomas $(n=220)$

\begin{tabular}{|c|c|c|c|c|c|}
\hline \multirow[t]{2}{*}{ Parameters } & \multirow[t]{2}{*}{$\mathrm{n}$} & \multicolumn{2}{|c|}{ Univariate analysis } & \multicolumn{2}{|c|}{ Multivariate analysis } \\
\hline & & $\begin{array}{l}\text { Mean survival time (months; } \\
95 \% \text { confidence interval) }\end{array}$ & P-value & $\begin{array}{c}\text { Hazard ratio } \\
\text { (95\% confidence interval) }\end{array}$ & P-value \\
\hline CIMP & & & 0.0007 & & 0.025 \\
\hline Negative & 156 & $86(82-90)$ & & Reference & \\
\hline Positive & 64 & $77(67-87)$ & & $2.249(1.105-4.574)$ & \\
\hline Age (years) & & & 0.0573 & & 0.966 \\
\hline 55 or less & 107 & $86(81-90)$ & & - & \\
\hline 56 or more & 113 & $85(78-92)$ & & $1.020(0.413-2.519)$ & \\
\hline$B R A F$ & & & 0.0781 & & 0.424 \\
\hline Wild & 193 & $83(79-87)$ & & - & \\
\hline Mutant & 26 & $77(61-94)$ & & $1.465(0.575-3.737)$ & \\
\hline AJCC staging & & & $<0.0001$ & & $<0.001$ \\
\hline Stage I and II & 153 & $89(86-92)$ & & Reference & \\
\hline Stage III & 59 & $76(66-87)$ & & $4.810(2.117-10.931)$ & \\
\hline Stage IV & 8 & $25(7-42)$ & & $23.855(8.653-65.764)$ & \\
\hline Differentiation & & & 0.0055 & & 0.080 \\
\hline Differentiated & 165 & $92(88-97)$ & & - & \\
\hline Poorly differentiated & 55 & $72(62-82)$ & & $1.899(0.927-3.892)$ & \\
\hline Crohn's-like lymphoid reaction & & & 0.0014 & & 0.171 \\
\hline Absent & 28 & $66(51-80)$ & & - & \\
\hline Mild or marked & 191 & $91(87-95)$ & & $0.561(0.245-1.283)$ & \\
\hline Peritumoral lymphocytic reaction & & & 0.0645 & & 0.289 \\
\hline Absent or mild & 168 & $83(78-88)$ & & - & \\
\hline Marked & 46 & $87(82-92)$ & & $0.518(0.154-1.746)$ & \\
\hline Chemotherapy & & & 0.7297 & & \\
\hline Not treated & 123 & $83(77-89)$ & & & \\
\hline Treated & 95 & $88(82-94)$ & & & \\
\hline
\end{tabular}

Abbreviations: MSI, microsatellite instability; CIMP, CpG island methylator phenotype; AJCC, American Joint Committee on Cancer.

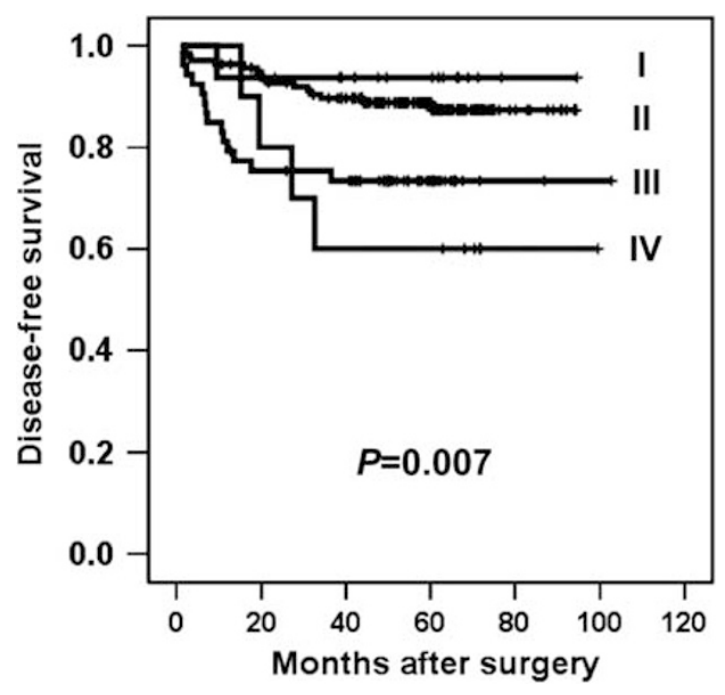

Figure 5 Kaplan-Meier curves of disease-free survival. The Kaplan-Meier survival analysis with log-rank test was performed for patients representing the following four subtypes of microsatellite-unstable colorectal cancers: $\mathrm{CpG}$ island methylator phenotype-low (CIMP-L)/0 MLH1 methylation-positive $(\mathrm{MLH} 1 \mathrm{~m}+)(\mathrm{I}, n=16), \mathrm{CIMP}-\mathrm{L} / 0 \mathrm{MLH} 1$ methylation-negative (MLH1m-) (II, $n=140)$, CIMP-H MLH1m + (III, $n=53)$, and CIMP-H MLH1m - (IV, $n=11)$.

\section{Discussion}

Our data clearly demonstrate the presence of microsatellite-unstable cases with discordant CIMP and MLH1 methylation status. We found that $17 \%$ of CIMP-H microsatellite-unstable tumors were MLH1m -, and 10\% of CIMP-L/0 microsatelliteunstable tumors were MLH1m + . We excluded the possibility that the discrepancy between CIMP status and MLH1 methylation status might be attributed to the technical errors of the MethyLight assay by performing the assay in triplicate using two different sets of MethyLight primers and probe that targeted the proximal and distal regions of MLH1 promoter. To exclude the possibility that the loss of the MSH2 protein in the CIMP-H MLH1m - subtype tumors might be caused by $\mathrm{MSH} 2$ promoter $\mathrm{CpG}$ island hypermethylation, we analyzed these tumors for methylation of MSH2 using the MethyLight assay, which revealed that none of the cases harbored MSH2 promoter CpG island hypermethylation (data not shown). This finding indicates that the aberrant hypermethylation induced by CIMP does not involve the MSH2 promoter $\mathrm{CpG}$ island locus. 
In our study, MLH1m + microsatellite-unstable tumors were heterogeneous in terms of clinicopathological and molecular features that correlated with CIMP status. Compared with the CIMP-H MLH1m + tumors, the CIMP-L/0 MLH1m + subtype tumors were associated with an earlier age of onset (53.2 vs 66.2 years, $P<0.001$, Student's $t$-test), reduced frequency of $B R A F$ mutation (6 vs $34 \%, P=0.027$, two-sided Fisher's exact test), a preponderance toward synchronous multiplicity (25 vs $0 \%$, $P=0.002$, two-sided Fisher's exact test), reduced predilection toward right colon (56 vs 85\%, $P=0.033$, two-sided Fisher's exact test), reduced frequency of nodal metastasis ( $13 \mathrm{vs} 40 \%, P=0.068$, two-sided Fisher's exact test), reduced frequency of poor differentiation (25 vs $43 \%, P=0.248$, twosided Fisher's exact test), and improved prognosis. Of the eight CIMP-panel markers, CACNA1G, IGF2, and NEUROG1 were not methylated in the CIMP-L/ 0 MLH1m + subtype tumors, in contrast to the increased methylation frequencies $(>90 \%)$ observed in the CIMP-H MLH1m + subtype tumors (Figure 3). These findings suggested that the CIMPL/0 MLH1m + and the CIMP-H MLH1m + subtype tumors are distinct entities and that the presence of MLH1 methylation does not necessarily correlate with the involvement of CIMP development of these microsatellite-unstable tumors.

The CIMP-L/0 MLH1m + subtype tumors were similar to those of the CIMP-L/0 MLH1m - subtype in several regards. Features shared in common include earlier age of onset, reduced predilection toward the anatomical subsite distribution to the right colon, and multiplicity within the large bowel. However, the CIMP-L/0 MLH1m + subtype was distinct from the CIMP-L/0 MLH1m - subtype in that it exhibited significantly different frequencies of loss of $\mathrm{MSH} 2$ and MLH1 expression. Furthermore, the patients of the CIMP-L/0 MLH1m + subtype did not succumb to death during the follow-up period after operation. Considering three features of the CIMP-L/0 MLH1m + subtype-the earlier age of onset, reduced number of methylated CIMP-panel markers $(n=1.125$, except for MLH1 methylation), and rare BRAF mutation rate-it is unlikely that this subtype arises from pre-existing serrated adenomas. Several studies have documented frequencies of $M L H 1$ methylation ranging from 0 to $55 \%$ in Lynch syndrome tumors, ${ }^{24-29}$ suggesting that an epigenetic mechanism might act as the second hit required to inactivate the wild-type allele in Lynch syndrome tumors. In Lynch syndrome tumors exhibiting MLH1 methylation, the number of methylated CIMP-panel markers has been demonstrated to be significantly lower than that of sporadic microsatellite-unstable tumors, ${ }^{11,25}$ indicating that the MLH1 methylation observed in the sporadic setting is a reflection of a widespread process of CIMP, whereas the MLH1 methylation in Lynch syndrome tumors is a more directed event.

In this study, we first reported the incidence of the CIMP-H MLH1m - tumor subtype at 5\% in micro- satellite-unstable colorectal cancers. This subtype can be seen in Wong et al's study (2 of 141 microsatellite-unstable colorectal cancers), although the presence of this subtype did not gain an attention. ${ }^{30}$ This subtype is characterized by increased incidence in male patients and a high frequency of loss of MSH2 expression. The average age of the patients with this subtype tended to be older than patients with the CIMP-L/0 MLH1m + or CIMP-L/0 MLH1m - subtype tumors. The CIMP-H MLH1m - subtype tumors are distinct from the CIMP-H MLH1m + subtype tumors in the following regards: (1) the male-to-female ratio was significantly different between the two subtypesthe CIMP-H MLH1m - subtype occurred predominantly in the male patients, whereas the CIMP-H MLH1m + subtype occurred predominantly in the female patients; (2) the KRAS/BRAF mutation rates were significantly different between these two subtypes-the BRAF mutation was predominantly detected in the CIMP-H MLH1m + subtype tumors, whereas the KRAS mutation was predominantly detected in the CIMP-H MLH1m - subtype tumors; and (3) the loss of MLH1 and MSH2 expression was different between these two subtypes-the loss of MLH1 expression was predominantly observed in the CIMP-H MLH1m + subtype tumors, whereas the loss of $\mathrm{MSH} 2$ was predominantly detected in the CIMP-H MLH1m - subtype tumors. To rule out the possibility that the loss of MSH2 expression in the CIMP-H MLH1m - subtype might be caused by MSH2 promoter $\mathrm{CpG}$ island hypermethylation, we analyzed the CIMP-H MLH1m - subtype tumors for methylation of MSH2 using the MethyLight assay, which revealed that none of the cases harbored MSH2 promoter CpG island hypermethylation (data not shown). Some of the clinicopathological features of the CIMP-H MLH1m - subtype tumors appeared similar to those of the CIMP-L/0 MLH1m - subtype tumors, including the increased frequency of loss of MSH2 expression, increased incidence in male patients, multiplicity, and rare $B R A F$ mutation rates. Considering that the CIMP-H MLH1m - subtype was associated with loss of MSH2 expression and rare BRAF mutations, we could not exclude the possibility that this subtype might represent Lynch syndrome tumors that acquired CIMP.

This study has limitations in that the information regarding the familial history of cases was not provided. Because this is a retrospective study, we could not obtain family histories from a considerable proportion of the cases. Furthermore, some family histories were so incomplete that we could not determine whether the respective cases satisfied the Amsterdam criteria II. Lastly, the genetic analysis of germline mutations of the DNA mismatch repair genes could not be performed because blood samples or fresh tissue samples were not procured from the patients.

It is well known that molecular features differ between right- and left-sided colon cancers. 
However, little information was available regarding whether there is a gradual change of molecular features along the long axis from the cecum to the rectum or an abrupt change of molecular features at the splenic flexure before the publication of Yamauchi et al's study results. Recently, Yamauchi et al. ${ }^{31}$ analyzed molecular features of colorectal cancers for their subsite distribution in the large bowel (cecum, ascending colon, hepatic flexure, transverse colon, splenic flexure, descending colon, sigmoid, rectosigmoid junction, and rectum) and found gradual decreases of CIMP-H and high-level microsatellite instability frequencies from the ascending colon to the rectum, but no abrupt change at the splenic flexure. However, our study demonstrated an abrupt decrease of high-level microsatellite instability frequency from the descending colon to the sigmoid. Discrepancy between findings of Yamauchi et al's study and those of our study might be attributed to the difference in the way of subsite division: we divided the large bowel into six subsites rather than nine subsites of Yamauchi et al's study and the descending colon in our study might include part of the splenic flexure, which might increase MSI-high frequency of the descending colon in our study. In contrast with Yamauchi et al's study in which cecal cancers showed lower frequency of MSI-high than that of ascending colon cancers, in our study, cecal cancers displayed higher frequency of MSI-high than that of ascending colon cancers.

In our study, colorectal cancer cases were bimodally distributed in terms of the number of methylated CIMP-panel markers (Supplementary Figure), but the two peaks were not separated as clearly as in Ogino et al's study. ${ }^{32}$ To explore the reason for the absence of clear separation of the two peaks, we performed the MethyLight assays of the eight markers in an independent set of colorectal cancer cases $(n=308)$ and determined the sensitivity and specificity of each CIMP-panel markers in the diagnosis of CIMP-H. All the eight markers showed high specificity (>94\%) for diagnosis of CIMP-H, but the sensitivity varied from 100 to $26 \%$ (Supplementary Table). When compared with the results of Ogino et al's study, ${ }^{32}$ our study showed higher specificities but lower sensitivities of individual markers, which might be related to the absence of clear separation of the two peaks.

A recent study that performed comprehensive genome-scale DNA methylation profiling of colorectal cancers has demonstrated that the MethyLightbased CIMP marker panel did not detect $21 \%$ of CIMP-H tumors identified by the Infinium DNA methylation data. ${ }^{33}$ However, all the CIMP-H tumors identified by the MethyLight-based marker panel belonged to CIMP-H cluster based on the Infinium DNA methylation data. The misclassification error of the MethyLight-based CIMP marker panel in detecting CIMP-H might affect the results of our study. Some of the CIMP-L/0 cases in our study might be CIMP-H based on the Infinium DNA methylation data. However, in our study, the CIMP-L/0 MLH1m + subtype showed rare BRAF mutation and younger age of onset, which are inconsistent with the features of CIMP-H colorectal cancers.

In conclusion, microsatellite-unstable colorectal cancers can be classified into four subsets depending on the combinatorial statuses of CIMP and MLH1 methylation, including CIMP-L/0 MLH1m - , CIMP$\mathrm{H}$ MLH1m +, CIMP-L/0 MLH1m +, and CIMP-H MLH1m - (in a descending order of frequency). Our study clearly demonstrates that CIMP + status and MLH1m + status do not completely overlap and that the discordant subtypes CIMP-H MLH1m - and CIMP-L/0 MLH1m + exhibit distinct clinicopathological and molecular features. These findings suggest that CIMP status, rather than MLH1 methylation status, is more likely to predict the clinicopathological features of microsatellite-unstable colorectal cancers.

\section{Acknowledgements}

This study was supported by a grant from the National R\&D Program for Cancer Control, Ministry of Health and Welfare, Republic of Korea (0720540), a grant from the Seoul National University Hospital Research Fund (0420120120), a grant from the Basic Science Research Program through the National Research Foundation of Korea (NRF) (20100007579), and the Mid-career Researcher Program through an NRF grant funded by the Ministry of Education, Science and Technology (MEST) (20110015646).

\section{Disclosure/conflict of interest}

The authors declare no conflict of interest.

\section{References}

1 Siegel R, Naishadham D, Jemal A. Cancer statistics 2012) CA Cancer J Clin 2012;62:10-29.

2 Ogino S, Goel A. Molecular classification and correlates in colorectal cancer. J Mol Diagn 2008;10:13-27.

3 Kang GH. Four molecular subtypes of colorectal cancer and their precursor lesions. Arch Pathol Lab Med 2011;135:698-703.

4 Boland CR, Goel A. Microsatellite instability in colorectal cancer. Gastroenterology 2010;138:2073-2087; e3.

5 Lynch HT, de la Chapelle A. Hereditary colorectal cancer. N Engl J Med 2003;348:919-932.

6 Pino MS, Mino-Kenudson M, Wildemore BM, et al. Deficient DNA mismatch repair is common in Lynch syndrome-associated colorectal adenomas. J Mol Diagn 2009;11:238-247.

7 Jass JR, Stewart SM. Evolution of hereditary nonpolyposis colorectal cancer. Gut 1992;33:783-786. 
8 Samowitz WS, Slattery ML. Microsatellite instability in colorectal adenomas. Gastroenterology 1997;112: 1515-1519.

9 Hawkins NJ, Ward RL. Sporadic colorectal cancers with microsatellite instability and their possible origin in hyperplastic polyps and serrated adenomas. J Natl Cancer Inst 2001;93:1307-1313.

10 Goldstein NS, Bhanot P, Odish E, et al. Hyperplasticlike colon polyps that preceded microsatellite-unstable adenocarcinomas. Am J Clin Pathol 2003;119:778-796.

11 Yamamoto $\mathrm{H}$, Min $\mathrm{Y}$, Itoh $\mathrm{F}$, et al. Differential involvement of the hypermethylator phenotype in hereditary and sporadic colorectal cancers with highfrequency microsatellite instability. Genes Chromosomes Cancer 2002;33:322-325.

12 Toyota M, Ahuja N, Ohe-Toyota M, et al. CpG island methylator phenotype in colorectal cancer. Proc Natl Acad Sci USA 1999;96:8681-8686.

13 Watanabe T, Kobunai T, Toda E, et al. Distal colorectal cancers with microsatellite instability (MSI) display distinct gene expression profiles that are different from proximal MSI cancers. Cancer Res 2006;66: 9804-9808.

14 Jass JR. HNPCC and sporadic MSI-H colorectal cancer: a review of the morphological similarities and differences. Fam Cancer 2004;3:93-100.

15 Jass JR, Walsh MD, Barker M, et al. Distinction between familial and sporadic forms of colorectal cancer showing DNA microsatellite instability. Eur J Cancer 2002;38:858-866.

16 Young J, Simms LA, Biden KG, et al. Features of colorectal cancers with high-level microsatellite instability occurring in familial and sporadic settings: parallel pathways of tumorigenesis. Am J Pathol 2001;159:2107-2116.

17 Bae JM, Kim MJ, Kim JH, et al. Differential clinicopathological features in microsatellite instability-positive colorectal cancers depending on CIMP status. Virchows Arch 2011;459:55-63.

18 Ogino S, Odze RD, Kawasaki T, et al. Correlation of pathologic features with $\mathrm{CpG}$ island methylator phenotype (CIMP) by quantitative DNA methylation analysis in colorectal carcinoma. Am J Surg Pathol 2006;30:1175-1183.

19 Kim JH, Shin SH, Kwon HJ, et al. Prognostic implications of $\mathrm{CpG}$ island hypermethylator phenotype in colorectal cancers. Virchows Arch 2009;455:485-494.

20 Dahlin AM, Palmqvist R, Henriksson ML, et al. The role of the CpG island methylator phenotype in colorectal cancer prognosis depends on microsatellite instability screening status. Clin Cancer Res 2010;16: 1845-1855.

21 Lee S, Cho NY, Choi M, et al. Clinicopathological features of $\mathrm{CpG}$ island methylator phenotype- positive colorectal cancer and its adverse prognosis in relation to KRAS/BRAF mutation. Pathol Int 2008;58: 104-113.

22 Eads CA, Danenberg KD, Kawakami K, et al. MethyLight: a high-throughput assay to measure DNA methylation. Nucleic Acids Res 2000;28:E32.

23 Ogino S, Kawasaki T, Brahmandam M, et al. Precision and performance characteristics of bisulfite conversion and real-time PCR (MethyLight) for quantitative DNA methylation analysis. J Mol Diagn 2006;8: 209-217.

24 Herman JG, Umar A, Polyak K, et al. Incidence and functional consequences of hMLH1 promoter hypermethylation in colorectal carcinoma. Proc Natl Acad Sci USA 1998;95:6870-6875.

25 McGivern A, Wynter CV, Whitehall VL, et al. Promoter hypermethylation frequency and BRAF mutations distinguish hereditary non-polyposis colon cancer from sporadic MSI-H colon cancer. Fam Cancer 2004;3:101-107.

26 Potocnik U, Glavac D, Golouh R, et al. Causes of microsatellite instability in colorectal tumors: implications for hereditary non-polyposis colorectal cancer screening. Cancer Genet Cytogenet 2001; 126:85-96.

27 Wheeler JM, Loukola A, Aaltonen LA, et al. The role of hypermethylation of the hMLH1 promoter region in HNPCC versus MSI+ sporadic colorectal cancers. J Med Genet 2000;37:588-592.

28 Cunningham JM, Christensen ER, Tester DJ, et al. Hypermethylation of the hMLH1 promoter in colon cancer with microsatellite instability. Cancer Res 1998;58:3455-3460.

29 Bettstetter M, Dechant S, Ruemmele P, et al. Distinction of hereditary nonpolyposis colorectal cancer and sporadic microsatellite-unstable colorectal cancer through quantification of MLH1 methylation by realtime PCR. Clin Cancer Res 2007;13:3221-3228.

30 Wong JJ, Hawkins NJ, Ward RL, et al. Methylation of the 3p22 region encompassing MLH1 is representative of the CpG island methylator phenotype in colorectal cancer. Mod Pathol 2011;24:396-411.

31 Yamauchi M, Morikawa T, Kuchiba A, et al. Assessment of colorectal cancer molecular features along bowel subsites challenges the conception of distinct dichotomy of proximal versus distal colorectum. Gut 2012;61:847-854.

32 Ogino S, Kawasaki T, Kirkner GJ, et al. Evaluation of markers for $\mathrm{CpG}$ island methylator phenotype (CIMP) in colorectal cancer by a large population-based sample. J Mol Diagn 2007;9:305-314.

33 Hinoue T, Weisenberger DJ, Lange CP, et al. Genomescale analysis of aberrant DNA methylation in colorectal cancer. Genome Res 2012;22:271-282.

Supplementary Information accompanies the paper on Modern Pathology website (http://www.nature.com/ modpathol) 